\title{
Analysis of an Indoor Biomedical Radar-based System for Health Monitoring
}

\author{
Marco Mercuri, Student Member, IEEE, Ping Jack Soh, Student Member, IEEE, Gokarna Pandey, \\ Peter Karsmakers, Guy A. E. Vandenbosch, Fellow Member, IEEE, Paul Leroux, Senior Member, \\ IEEE, and Dominique Schreurs, Fellow, IEEE
}

\begin{abstract}
Innovative technology approaches have been increasingly investigated for the last two decades aiming at human being long-term monitoring. However, current solutions suffer from critical limitations. In this paper, a complete system for contactless health-monitoring in home environment is presented. For the first time, radar, wireless communications, and data processing techniques are combined together enabling contactless fall detection and tag-less localization. Practical limitations are considered and properly dealt with. Experimental tests, conducted with human volunteer in a realistic room setting, demonstrate an adequate detection of the target's absolute distance and a success rate of $94.3 \%$ in distinguishing fall events from normal movements. The volunteers were free to move in the whole room with no constraints in their movements.
\end{abstract}

Index Terms-Fall detection, health monitoring, radar remote sensing, tag-less localization, Zigbee communication.

\section{INTRODUCTION}

$\mathrm{T}$ HE senior citizen population of older than 60 years has been steadily increasing worldwide. This situation has resulted in a growing need for novel assistive technologies that enable routine long-term home monitoring [1]. Conventional approaches are based on devices attached to the patient's body, involving pressing a button, e.g., worn as a necklace, in emergency situations [2], [3]. However, persons in such situation may already be unconscious or no longer reflexive enough to do so. The ideal solution is therefore a contactless health monitoring system, avoiding the need for actions by the elderly person.

In the last two decades, attention has been focused mainly on contactless vital signs monitoring [4]. These academic developments are based on radar techniques implemented as a single device sensor, e.g., Continuous-Wave (CW) Doppler radar [5] or Ultra-Wide-Band Impulse-Radio (UWB-IR) radar

Manuscript received October 15, 2012. This work was supported by FWOFlanders and KU Leuven GOA project.

The authors are with the Department of Electrical Engineering ESAT, Catholic University of Leuven, Leuven, Belgium (phone: +32 16 321820; fax: +32 16 321063; e-mail: Marco.Mercuri@esat.kuleuven.be).

P. J. Soh is also with School of Computer \& Communication Engineering, Universiti Malaysia Perlis, Perlis, Malaysia.

P. Karsmakers and P. Leroux are also with the Thomas More Kempen, Geel, Belgium.
[6]. The capabilities of such devices are limited to heartbeat and respiration rate monitoring under "ideal conditions", e.g., constrained to a single motionless person (i.e., seated or lying down). In recent years, remote fall detection has become a primary interest in connection to health monitoring in home environments [7]-[9]. Instances of such investigations are systems based on video cameras [10], floor vibration, and acoustic sensors [11], [12]. Besides privacy concerns, video camera systems are still troubled by issues related to low light environments, field of view, and image processing, resulting in a success rate of $90 \%$ using two cameras [10]. Successes in floor vibration and acoustic sensors are similarly limited due to environmental interference and background noise. Moreover, they are also less effective in detecting cases of "soft" human falls, defined as a fall after the individual collides with an object (table, chair, carpet, etc.) [11], [12].

Due to the disadvantages of existing fall detection technologies, there is a need for further solutions. An alternative approach based on radar techniques has been demonstrated by the authors [13], [14]. The approach is able to detect fall events and to localize a person without the need for a radio frequency identification (RFID) tag on the person. However, to satisfy the spectrum mask requirements and to have a practical device, traditional radar architectures are not suitable solutions for this application and the waveform to be transmitted needs to be properly designed [15].

In this work, a full system is proposed enabling indoor, noninvasive fall detection and tag-less localization. It combines radar, wireless communications, and data processing techniques. Moreover, it has been designed to satisfy the European and Federal Communications Commission (FCC) UWB spectrum masks, and it can also be potentially connected to medical monitoring personnel to provide a prompt alert in the event of emergencies.

In Section II the monitoring principle and underlying theory are presented. The sensor architecture is explained in Section III, and the experimental results are discussed in Section IV.

\section{OPERATIONAL PRINCIPLE}

Fig. 1 shows a simplified block diagram of the proposed health monitoring system. It consists of a sensor, combining both radar and wireless communications features, and a base 
station for data processing. A radar waveform is generated and sent to the target, and then its reflected echo, containing speed and absolute distance information, is collected by the receiver. The resulting baseband signals are digitized and transmitted wirelessly to a base station that consists of a Zigbee module, a laptop, and a microcontroller. The latter collects and transfers the data received of the Zigbee module to the laptop to determine remotely the target's absolute distance and to distinguish a fall event from normal movements (e.g., walking, sitting down). The data processing is not performed by the sensor in order to avoid complex processor on board, reducing costs, size, and also energy consumption. Moreover, this represents a flexible solution if multiple sensors will be used in the future. In fact, the base station should combine and process multiple information at the same time.

The radar waveform is based on a hybrid approach presented by the authors in [15]. It consists of a single tone, at $f_{I S M}=5.8 \mathrm{GHz}$ in the ISM band, alternated with a stepped frequency continuous wave (SFCW) waveform working in the UWB band, as shown in Fig. 2. Each tone lasts $1 \mathrm{~s}$ and it is used to continuously detect the speed of a person using the Doppler concept. The SFCW waveform is used to detect the target's absolute distance. It consists of $N=40$ coherent CW pulses (called burst) whose frequencies are increased from pulse to pulse by a fixed increment $\Delta f=25 \mathrm{MHz}$. Each pulse is $T=50 \mu$ s long, resulting in a burst duration $N \cdot T$ of $2 \mathrm{~ms}$, while its total band $N \cdot \Delta f$ is $1 \mathrm{GHz}$ positioned between 6 and $7 \mathrm{GHz}$, enabling a smallest resolution of $15 \mathrm{~cm}$. The full waveform is 1.002 seconds. In comparison to [15], we introduce some changes in subsection B to tackle some practical issues.

In this section, the waveform spectral analysis is first discussed. Next, these considerations are used to justify the choice of the waveform parameters, and to explain the operations of compensation and calibration in data processing. Moreover, the technique to distinguish fall events from normal movements is also described.
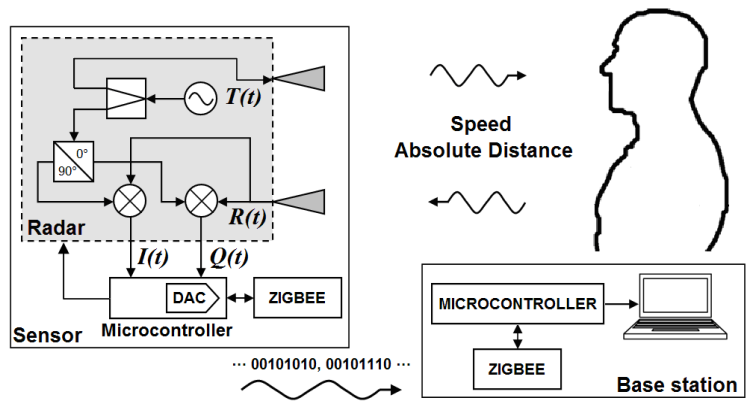

Fig. 1. Simplified block diagram of the health monitoring system.

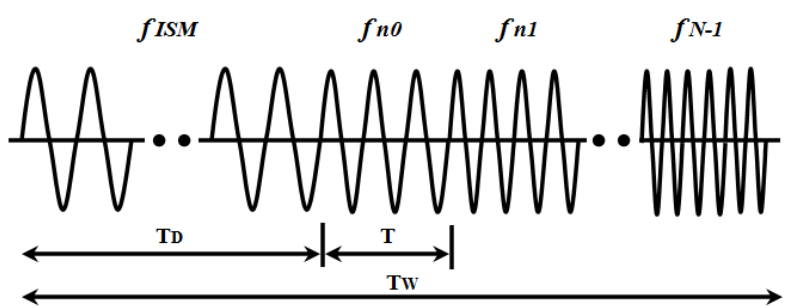

Fig. 2. Designed radar waveform.

\section{A. Spectral Analysis}

Neglecting the initial amplitudes, the transmitted waveform can be expressed as:

$T_{s}(t)=\cos \left[2 \pi f_{I S M} t+\varphi_{I S M}(t)\right]$

for $0 \leq t \leq T_{D}$, and

$T_{S F C W}(t)=\cos \left[2 \pi\left(f_{0}+n \Delta f\right) t+\varphi_{n}(t)\right]$

for $T_{D}<t \leq T_{W}$, with $0 \leq n<N$. If the waveform is reflected by a target at a distance $D$, the received signal will then be represented as:

$R_{s}(t)=\cos \left[2 \pi f_{I S M}\left(t-\frac{2 D}{c}\right)+\varphi_{I S M}\left(t-\frac{2 D}{c}\right)\right]$

and

$R_{S F C W}(t)=\cos \left[2 \pi\left(f_{0}+n \Delta f\right)\left(t-\frac{2 D}{c}\right)+\varphi_{n}\left(t-\frac{2 D}{c}\right)\right]$

The output of the IQ mixer (Fig. 1) can be modeled as the product of the received signal with a copy of the transmitted signal followed by a lowpass filter. For a quadrature sampling, it is given as:

$e^{-j \phi_{i}}=I(t)+j Q(t)$

with

$\phi_{i}=\phi_{s}=2 \pi f_{I S M} \frac{2 D}{c}+\theta_{I S M}+\Delta \varphi_{I S M}(t)$

for the single tone, and

$\phi_{i}=\phi_{n}=2 \pi\left(f_{0}+n \Delta f\right) \frac{2 D_{n}}{c}+\theta_{n}+\Delta \varphi_{n}(t)$

for the SFCW signal, where

$\Delta \varphi(t)=\varphi(t)-\varphi\left(t-\frac{2 D}{c}\right)$

is the residual phase noise, which is negligible, while $\theta$ is the contribution of the phase shift at the target surface (approximately $180^{\circ}$ ) plus additional phase difference between the mixer and the antenna. Only one sample for pulse width $T$ has been considered. In the case of a moving target, the range $D$ can be written as:

$D=D_{I S M}+v(t) t$ 
for $0 \leq t \leq T_{D}$, and as

$D_{n}=D_{0}+v\left(t_{n}\right) n T$

for $T_{D}<t \leq T_{W}$, and $D_{I S M}$ and $D_{0}$ are defined as the ranges to the target at the particular time $t=0$ and $t=T_{D}$, respectively. Combining eqs. (9) and (10) with eqs. (6) and (7), the phase of the baseband signal becomes:

$\phi_{s}=2 \pi f_{I S M} \frac{2 D_{I M S}}{c}+2 \pi \frac{2 f_{I S M} v(t)}{c} t+\theta_{I S M}$

and

$$
\begin{aligned}
\phi_{n}=\frac{4 \pi f_{0} D_{0}}{c} & +2 \pi \frac{\Delta f}{T} \frac{2 D_{0}}{c} n T+ \\
& +2 \pi \frac{2 v\left(t_{n}\right) f_{0}}{c} n T+2 \pi \frac{2 v\left(t_{n}\right) n \Delta f}{c} n T+\theta_{n}
\end{aligned}
$$

The last two equations are essential in the waveform design and for data processing, which consequently determines the hardware requirement and system complexity. Eq. (11) represents that the signal is proportional to the target's speed, and it dictates also its sampling rate. On the other hand, eq. (12) is used to determine the target's absolute distance. In particular, the first two terms represent the case of a stationary target since they are not influenced by speed, $v(t)$. The first term represents a constant phase shift, which is not of any practical significance, while the second term is a multiplication between the rate of frequency change $\Delta f / T$ and the signal round-trip time $2 D / c$. The latter term provides the distance information. The remaining terms are undesirable. The third term represents the Doppler frequency shift due to target motion, which adds to the frequency shift of the second term, resulting in a shift of target distance from its true value. The fourth term exists due to the interaction of the frequencyvarying step waveform with the target's motion, resulting in a spread of the target peak. Compensating these effects is difficult due to the unknown instantaneous target velocity. The final term is frequency-dependent, which adds a fixed frequency shift to the actual target distance.

\section{B. Waveform Design}

The proper choice of the parameters $T, T_{D}$, and $N$ allows to satisfy the spectrum masks while simultaneously having sufficient transmit power to track a person in a typical room setting.

The waveform has been designed to monitor continuously the speed of the target and to determine its absolute distance every $T_{D}=1 \mathrm{~s}$. Assuming a maximum target movement/falling speed of $2 \mathrm{~m} / \mathrm{s}$, the I/Q baseband signals containing speed information should be acquired at least with a sample rate of about $6.5 \mathrm{~ms}$. This value is defined considering the Nyquist theorem and eqs. (5) and (11), where $f_{I S M}$ is $5.8 \mathrm{GHz}$. Experimental evaluations on human volunteers have failed to demonstrate significant improvements even when the sample time is decreased below $7.2 \mathrm{~ms}$. This indicates that the maximum speed produced by the subjects' movements is about $1.8 \mathrm{~m} / \mathrm{s}$, lower than the $2 \mathrm{~m} / \mathrm{s}$ initial assumption. However, a sample rate of $4 \mathrm{~ms}$ has been chosen providing a sufficient margin to detect movements of humans with different weights.

To avoid losses in speed samples and to simultaneously determine the target's corresponding distance, the $N \mathrm{CW}$ pulses are sent, every one second, in between speed samples. This involves a maximum burst interval $N \cdot T$ of $4 \mathrm{~ms}$. In order to have an unambiguous range of $5 \mathrm{~m}$, the increment $\Delta f$ from the second term of eq. (12) should be fixed to $30 \mathrm{MHz}$. This theoretical value cannot be used in practice since the third and fifth terms shift the target position outside the unambiguous range. The third, and consequently the fourth term, can be neglected by properly choosing the burst duration $N \cdot T$ such that the target is considered static during this interval, i.e., speed $v(t)=0$. The burst interval of $2 \mathrm{~ms}$ is sufficiently short to consider the static target assumption true. Moreover, this ensures also that the downconverted signals of the SFCW waveform consist of I/Q direct current (DC) levels. This means that only two samples can be acquired per pulse width $T$ fixing the sample time of SFCW waveform to $50 \mu \mathrm{s}$.

On the contrary, the fifth term, which is not negligible, is compensated through a calibration procedure, resulting, for the presented sensor, in about $1 \mathrm{~m}$ fixed shift from the correct target distance. These considerations resulted in $\Delta f$ to be fixed at $25 \mathrm{MHz}$ to enable a $6 \mathrm{~m}$ coverage, $5 \mathrm{~m}$ for the desired target range and another $1 \mathrm{~m}$ allowance to cater for the fixed shift.

\section{Data Processing}

The digitized I/Q baseband signals are processed remotely in the base station using Matlab. The samples are first related as in eq. (5), and then split as according to category to be processed separately, namely whether the samples serve for speed monitoring or for absolute distance detection.

A movement classification based on a Least Square Support Vector Machine (LS-SVM) approach [16] is applied to analyze the speed samples in order to distinguish falls from normal movements. The key relies on the different changes in speed experienced during a fall or a normal movement. During a fall, in fact, the speed continuously increases until the sudden moment when the fall stops abruptly. During a normal movement, the Doppler signal experiences a controlled movement. More precisely, while a person is sitting or lying down, the speed first gradually increases, and then decreases to a smooth stop, whereas during a walk, instead, the speed is quite constant over time. The acquired speed signals, or activities, are used to build a data set. Before learning a model, the raw radar data is preprocessed. Each activity is grouped in a segment of 2 seconds, considered sufficient to cover the details of the activities. Given such segments, the data is then transformed using Fast Fourier Transform (FFT) from which only the magnitude spectrum is retained. Two alternatives have been considered, namely 1) to compute the FFT directly on the complete segment and 2) to use the Short Time FFT (STFT). 
In the latter case, the segment is first chopped into $50 \%$ overlapping frames which are each multiplied with a Hamming window after which the FFT is computed on each of these frames. As opposed to the FFT, the STFT can represent time dependent structures and results in higher performance in case of signals that experience a gradual change in velocity. In the LS-SVM framework a choice concerning a kernel function must be made. A kernel function can be seen as a similarity function which defines how signals are compared. For the first option, the LS-SVM is used in combination with a linear and Radial Basis Function (RBF) kernel on the FFT data, while to handle the STFT data, the LS-SVM is combined with the Global Alignment (GA) kernel. Prior to the learning phase, the data was standardized such that each dimension has zero mean and unit standard deviation.

The target's range profile is determined applying the Inverse Fast Fourier Transformer (IFFT). However, this operation has as main challenge the distinction of the target's reflection from the effects of backscattering and crosscoupling between the two antennas. The latter involve strong reflections that overwhelm the much weaker reflected/received signal, resulting in the inability to acquire any meaningful target information. Besides, the presence of cluttering due to furniture in a practical environment must also be considered. Both factors can be eliminated by a compensation that consists in determining an environmental range profile, characterizing the total contribution from both cross-coupling and cluttering. Its magnitude is then subtracted from the range profiles obtained with the target in the room. Then, the range profile is shifted by a fixed value to compensate for the effect of the fifth term in eq. (12). This value is obtained through calibration using a flat metal plate, placing it at a well-known distance from the antennas to evaluate its range profile. The value of its corresponding peak is read and subtracted from this calibrated distance, thus yielding $\theta_{n}$.

However, it has to be noted that this operation can be only successful if the backscattering and crosstalk are strongly reduced, as explained in the next section.

\section{SENSOR ARCHITECTURE}

Fig. 3 shows the overall sensor block. It consists of three main parts, namely the radar module, the AT86RF231 Zigbee module, and the microcontroller. The sensor block is combined with two-element dual-band antennas, designed to operate at both the radar and Zigbee ISM frequency bands. As will be explained in subsection $B$, the main challenge is to reduce the backscattering and crosstalk effects while presenting also a semispherical radiation pattern. No off-theshelf antennas are available dealing with such challenges. A wideband circulator with one antenna could represent a more compact solution. However, although it does not experience the cross talk, the mismatch between the antenna and its feed line involves a strong reflection that overwhelms the reflected signal. This effect cannot be reduced below a practical value (i.e., $-30 \mathrm{~dB}$ ) in the whole radar bandwidth as it was possible with the cross talk in the case of two antennas.

The microcontroller controls the RF switch to connect alternately the radar transmitter and the Zigbee module to the transmitter antenna, labeled $T X$ in Fig. 3. However, the latter is used by the Zigbee module both to transmit and to receive frames. In fact, the sensor may receive commands from the base station. This happens to synchronize base station and sensor after power-on.

Batteries provide the power supply to the sensor.

We will now detail the radar module and the antenna design. As the Zigbee module is an off-the-shelf component, it is not further described.

\section{A. Sensor}

The radar module integrates a Fractional-N PLL with a wideband VCO, a $5.8-7 \mathrm{GHz}$ wideband Wilkinson power divider, an RF switch, a low noise amplifier (LNA), a gain block, an In-phase and Quadrature (IQ) mixer, and baseband filters and amplifiers. The microcontroller programs the synthesizer of the PLL to generate the radar waveform, described in Section II.B, with a maximum transmit power level of $+2 \mathrm{dBm}$. A $5.8 \mathrm{GHz}$ single tone is produced at every $1 \mathrm{~s}$ interval, immediately followed by this SFCW waveform. The sensor requires about $30 \mu$ s to generate a new frequency, by which the first $20 \mu$ s is to program the PLL, while another 8 $\mu$ s is to take into account the VCO maximum settling time. The combined LNA and gain block is chosen to provide a total gain of about $30 \mathrm{~dB}$ to avoid saturation of the gain block. This is considering that the two antennas present a cross-coupling of about $-35 \mathrm{~dB}$ within the operation bandwidth and that the target could move very close to the antennas, producing a high reflection and thus high received power.

The bipolar I/Q baseband signals are then acquired and digitized by an ADC inside the microcontroller. Since it works with unipolar signals, between 0 and $3.2 \mathrm{~V}$, the outputs of the mixer are bandpass filtered, amplified and then a $1.6 \mathrm{~V} \mathrm{DC}$ level is added to position the signals to the center of ADC's

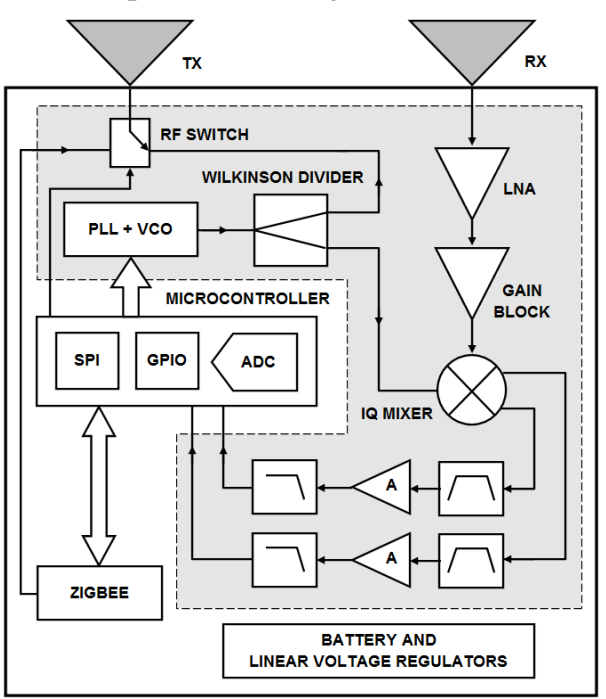

Fig. 3. Block diagram of the sensor. 
dynamic range. The lowpass filter serves both as an antialiasing filter and as a charge reservoir for the ADC's switched capacitor input stage.

The 10-bit ADC requires about $20 \mu$ s to acquire the samples and to digitize the two I/Q components before the next frequency can be generated. These acquired and digitized samples, containing the target's information, are then sent to the base station. This transmission must be properly managed while simultaneously acquiring the monitoring signals. Since the Zigbee protocol only transmits frames containing bytes, each of the acquired 10 bit samples should be ordered into two bytes. However, since the I and Q samples are acquired at the same time, they can be mapped in 3 bytes. Sampling the signal containing speed information at $250 \mathrm{~Hz}$ for a duration of $1 \mathrm{~s}$, and considering the I/Q components, the sensor is expected to accumulate 502 samples. This translates into 753 bytes. The transmission of these frames must be executed in between speed sampling instants. For that reason, the speed samples are mapped in frames of 75 bytes, 25 I samples and 25 Q samples. The transmission of such frames requires about $2.6 \mathrm{~ms}$ each, much shorter than the duration of the acquisition period. Each frame is therefore filled after $100 \mathrm{~ms}$ and then transmitted before the next new sample is acquired. However, the transmission of the last 3 speed bytes is achieved with the transmission of the 80 I/Q burst samples, i.e. 120 bytes. This involves the transmission of two consecutive frames, each with 63 and 60 bytes, respectively. The former, containing also the last 3 speed bytes, is transmitted immediately after the acquisition of the first new I/Q speed, while the latter is transmitted after the acquisition of the second I/Q speed samples.

The $5.8 \mathrm{GHz}$ tone is generated immediately after the SFCW waveform. However, the first I/Q speed samples are acquired after about $2 \mathrm{~ms}$ after the acquisition of the last burst samples. This is done to hold the $4 \mathrm{~ms}$ speed sampling time as a constant.

\section{B. Antenna}

Regarding the antennas for the sensor, two identical dualband antennas based on the coplanar waveguide (CPW) bowtie have been designed for the sensor. According to the system requirements, the antennas are to be used for target monitoring between 5.8 and $7 \mathrm{GHz}$, and for sensor-to-base station communication at $2.45 \mathrm{GHz}$. Besides resonance prerequisites, a set of stringent design requirements is imposed, which includes size compactness and radiation characteristics. Firstly, the backscattering within the frequency band where the sensor is used to locate the target and to determine its speed must be strongly reduced to enable maximum forward power towards the target. A failure in doing so will obviously limit the forward transmit power, resulting in a weak reflection from the target, and in a waste of energy. Moreover, since the sensor is intended to be mounted either to the wall or to the ceiling, the relative reflection of the wall or ceiling will bury the much weaker target's reflection.
Another challenge that arises when attempting to arrive at a compact system size is the problem of the inter-element antenna cross-coupling. As was explained earlier, such large cross-coupling enables the power "overflow" from the transmitting antenna to the adjacent receiving element involving a strong reflection. These two undesired effects therefore involve the decrease of the total receiver's gain to avoid the saturation both of the amplifiers and of the ADC. Thus, the longer the distance to the target is, the weaker is the reflection such that it is no longer perceptible by the ADC's resolution, as it is buried in the noise.

These considerations, plus the objective for a $5 \times 5 \mathrm{~m}$ equivalent room area coverage lead to the following design requirements: (1) a compact antenna footprint, approximately equivalent to the overall sensor's size; (2) an antenna beamwidth of approximately $60^{\circ}$ for a wide coverage in the azimuth plane; and (3) a minimum gain of approximately $6 \mathrm{~dB}$ with unidirectional forward radiation.

The designed antennas are shown in Fig. 4. Each bow-tie element is fabricated on a $1.524 \mathrm{~mm}$ thick Rogers RO4003 substrate, with a relative permittivity $\varepsilon_{r}$ of 3.38 and loss $\operatorname{tangent} \tan \delta$ of 0.021 . Two identical antennas are then secured onto $18.5 \mathrm{~mm}$ thick foam substrates with $\varepsilon_{r}=1.05$ prior to placement on a common ground plane sized at 112 x $42 \mathrm{~mm}$. Besides minimizing antenna back radiation, such arrangement enables bandwidth broadening across 6 to $7 \mathrm{GHz}$. The closeproximity placement of the antennas are almost certain to induce cross-coupling, thus a $39 \times 31 \times 1 \mathrm{~mm}$ vertical wall is introduced between the two antennas, placed $19.5 \mathrm{~mm}$ from each element.

The designed antenna combination has successfully achieved its intended purpose. In fact, the use of the common ground plane reduces strongly the backscattering, while the use of the metal wall suppresses the cross-coupling $\left(S_{21}\right)$ besides maintaining a satisfactory reflection coefficient $\left(\mathrm{S}_{11}\right)$, as shown in Fig. 5. For this fabricated prototype, the $S_{11}$ results between $6.6 \mathrm{GHz}$ and $7 \mathrm{GHz}$ are indeed slightly above $-10 \mathrm{~dB}$.

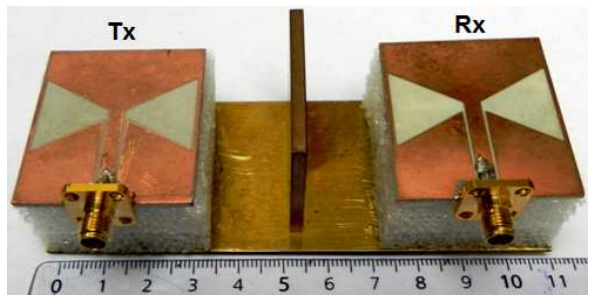

Fig. 4. Fabricated prototype of the proposed two-element bow-tie antenna.

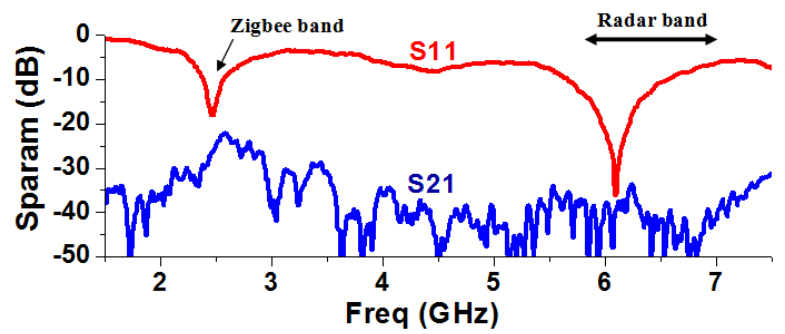

Fig. 5. Measured antenna S-parameters. 
However, the highest/worst $\mathrm{S}_{11}$ within this range is $-7 \mathrm{~dB}$, which translates to a maximum of $20 \%$ reflected RF power. This is mainly due to both the soldering and the difficulty of realizing a completely flat foam spacer. Similarly for the system, this means that a maximum of $20 \%$ less power is transmitted, resulting in a lower target-reflected signal detected by the receiver. This may produce an error in absolute distance detection when the target is really far away from the antenna, i.e., more than $5 \mathrm{~m}$. At these distances, reflections from the target received within the $6.6-7 \mathrm{GHz}$ band will not be perceptible by the receiver despite the sufficient signal amplitudes received in the $6-6.6 \mathrm{GHz}$ band. This then potentially causes a wrong target location interpretation in the range profile. However, the measurements indicated this is not a problem in a $5 \times 5 \mathrm{~m}^{2}$ sized room.

\section{EXPERIMENTAL RESULTS}

Experimental evaluations have been conducted with real human volunteers who are allowed to move freely in the whole room. The sensor has been fixed to the wall at $1.5 \mathrm{~m}$ of height. Furniture and metallic shelves are deliberately included to enable the existence of clutter and reflections, mimicking a typical room setting. Falls are mimicked with two different human volunteers, with a similar $1.75 \mathrm{~m}$ height but different weights, to enable the evaluation of different fall speeds. The first and second subjects' weighs are $90 \mathrm{~kg}$ and $75 \mathrm{~kg}$, respectively. However, only frontal falls have been evaluated at different locations at radial distances in the whole room, using an inflated mattress to avoid injuries. Also, only one person was present in the room at a time.

The prototype implementation of the proposed system is proven to be accurate as illustrated in the following results.

\section{A. Tag-less localization}

Fig. 6a represents an initial range profile of a person at $4 \mathrm{~m}$ away from the antennas, prior to the compensation and calibration process. The peak, which is supposed to indicate the target's absolute distance, is totally overwhelmed by the undesired reflections originating from the clutter and the antenna's cross-coupling. In particular, the latter is the most dominant effect as emphasized by the strong peak at $90 \mathrm{~cm}$. This indicates also the position of the two antennas in the range profile, which in theory should be at $0 \mathrm{~m}$. The peak therefore results shifted in range due to the effect of the fixed frequency shift $\theta_{n}$, whose value can be read and used during calibration.

After applying the compensation and the calibration steps, the target's peak can be perfectly distinguished as shown in Fig. 6b.

However, it should be noted that the radar has a range resolution of $15 \mathrm{~cm}$. It means that any target's physical distance will be rounded to the nearest location/resolution provided by the radar. This also establishes the maximum error in localization of $7.5 \mathrm{~cm}$.

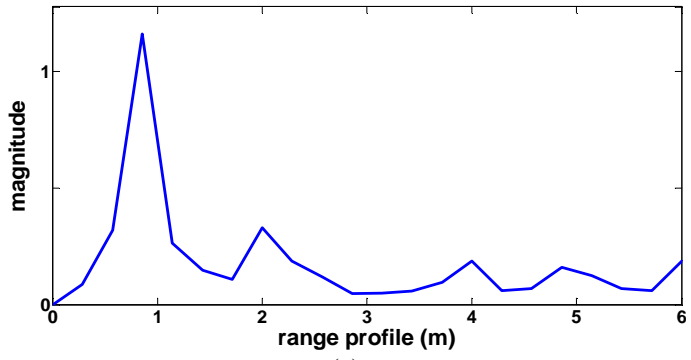

(a)

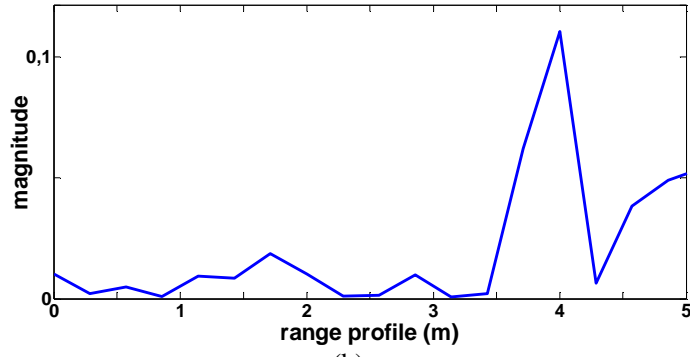

(b)

Fig. 6. Range profile of a target at $4 \mathrm{~m}$ away the antennas before (a) and after (b) compensation and calibration.

\section{B. Fall detection}

A data set was built containing 70 activities measured from two persons. In particular, 20 walking signals have been acquired for each person, who was allowed free movement in the whole room, and 30 fall signals have been acquired with each subject located at known distances from the antennas. Figs. $7 \mathrm{a}$ and $7 \mathrm{~b}$ show the speed signals respectively during a walking and a fall movement. The LS-SVM model is trained using the data of a single person (target 1) and then validated using the data from the other person (target 2). This process is repeated two times since data from two persons was available. The results of the classification are shown in Table I. It can be seen that the GA kernel, that incorporates time dependent information, presents a success rate in distinguishing fall events from normal movements of $94.3 \%$ outperforming the linear and RBF kernels. This is expected since falls exhibit a time dependent structure as the speed increases continuously until the sudden moment when it stops abruptly. An alternative method called Dynamic Time Warping (DTW) combined with a Euclidean distance measure is frequently used to classify sequences of vectors. In order to compare the LS-SVM with GA solution to this standard method an additional experiment was included. The results show that the GA kernel slightly outperforms the DTW alternative on this data set.

By reducing further the crosstalk, it will be possible to increase the total receiver gain and consequently to improve the accuracy of velocity detection, besides the detection range extension. Also the system's sampling rate influences the accuracy of the speed detection, i.e., the higher it is, the more accurate is its detection. However, this will significantly complicate the system since the duration of the SFCW pulses must be shortened and the ADC sample rate increased. This will involve higher power consumption and a larger number of transmitted frames to the base station. However, it should be noted that in this application the main goal is to detect the changes in speed and not to determine how accurate is the value of the instantaneous speed. 


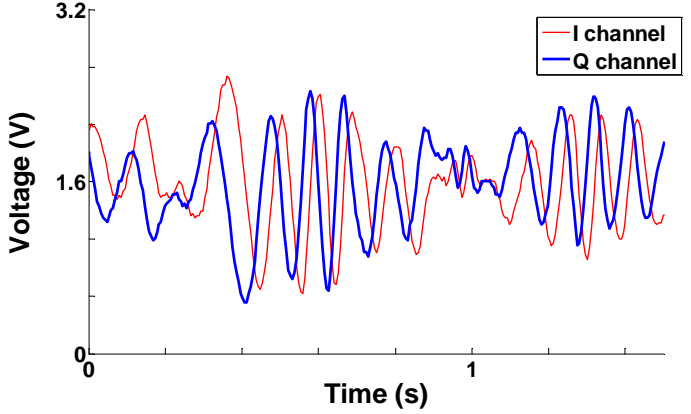

(a)

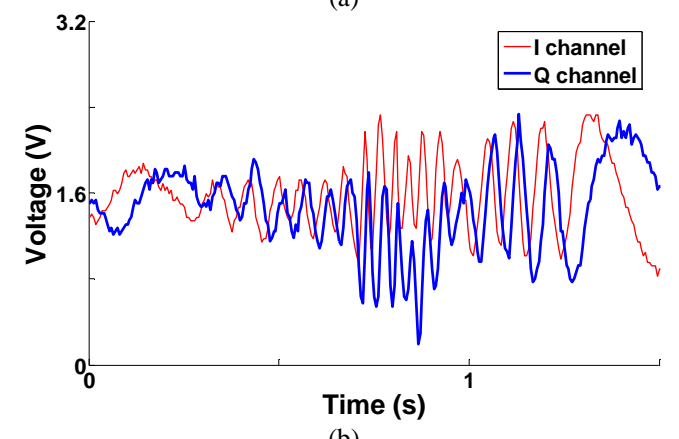

(b)

Fig. 7. Speed signal during (a) a walking movement and during (b) a fall event. The frequency of the signal is proportional to the radial velocity of the person during the movement. An inflating mattress has been used when invoking falls. For that reason, the corresponding speed signals do not stop suddenly but there is also the effect of the rebounds on the mattress.

TABLE I

FALSE POSITIVES AND SUCCESS RATE USING FOUR CLASSIFICATION MODELS

\begin{tabular}{ccccc}
\hline \hline \multirow{2}{*}{$\begin{array}{c}\text { Classification } \\
\text { model }\end{array}$} & $\begin{array}{c}\text { \% False } \\
\text { Positives }\end{array}$ & $\begin{array}{c}\text { \% Success } \\
\text { Rate }\end{array}$ & \% False & \% Success \\
& 15 & 85.7 & 10 & 82.9 \\
\hline LS-SVM lin & 15 & 80 & 15 & 85.7 \\
LS-SVM RBF & 0 & 94.3 & 0 & 94.3 \\
LS-SVM GA & 10 & 91.4 & 0 & 94.3 \\
DWT &
\end{tabular}

\section{CONCLUSION}

A radar-based system has been proposed as a new approach for contactless fall detection and tag-less localization in indoor environment. This is inline with the growing need for novel health care solutions. The full system combining radar, wireless communications, and data processing techniques has been analyzed and described. The prototype implementation has been designed to satisfy the European and Federal Communications Commission (FCC) UWB spectrum masks. Practical problems, such as backscattering and crosstalk, have been also addressed. Experimental evaluations with real human subjects have demonstrated accurate detection of the target's absolute distance and fall events.

Next step is to combine multiple sensors in a wireless sensor network configuration, in order to monitor multiple persons and to increase the accuracy and coverage area, beyond one room. The final application is automated remote monitoring in private homes, although it can also be adopted for nurse call solutions in nursing homes.

\section{REFERENCES}

[1] O. Boric-Lubecke and V.M. Lubecke, "Wireless house calls: using communications technology for health care and monitoring," IEEE Microwave Magazine, vol. 3, pp. 43-48, Sep. 2002.

[2] E. Kantoch, J. Jaworek, and P. Augustyniak, "Design of a wearable sensor network for home monitoring system," Federated Conference on Computer Science and Information Systems (FedCSIS), pp. 401-403, 18-21 Sept. 2011.

[3] V.M. Lubecke, and O. Boric-Lubecke, "Wireless technologies in sleep monitoring," IEEE Radio and Wireless Symposium, pp. 135-138, 18-22 Jan. 2009.

[4] L. Changzhi, J. Cummings, J. Lam, E. Graves, and W. Wenhsing, "Radar remote monitoring of vital signs," IEEE Microwave Magazine, vol. 10, no. 1, pp. 47-56, Feb. 2009.

[5] A. D. Droitcour, O. Boric-Lubecke, V.M. Lubecke, J. Lin, and G.T.A. Kovac, "Range correlation and I/Q performance benefits in single-chip silicon Doppler radars for noncontact cardiopulmonary monitoring," IEEE Trans. Microwave Theory Tech., vol. 52, no. 3, pp. 838-848, Mar. 2004.

[6] I.Y. Immoreev, S. Samkov, and T.-H. Tao, "Short-distance ultra wideband radars," IEEE Aerospace and Electronic Systems Magazine, vol. 20, no. 6, pp. 9-14, June 2005.

[7] S. R. Lord, C. Sherrington, and H. B. Menz, Falls in Older People: Risk Factors and Strategies for Prevention. Cambridge, U.K., Cambridge Univ. Press, 2007.

[8] Y. Xinguo, "Approaches and principles of fall detection for elderly and patient," $10^{\text {th }}$ International Conference on e-health Networking, Applications and Services (HealthCom), pp. 42-47, Singapore, 7-9 July 2008.

[9] N. Noury, A. Fleury, P. Rumeau, A.K. Bourke, G.O. Laighin, V. Rialle, and J.E. Lundy, "Fall detection - Principles and Methods," $29^{\text {th }}$ Annual International Conference of the IEEE Engineering in Medicine and Biology Society, pp. 1663-1666, Lyon, France, 22-26 Aug. 2007.

[10] M. Yu, S. M. Naqvi, and J. Chambers, "A robust fall detection system for elderly in a smart room," International Conference on Acoustics Speech and Signal Processing, pp. 1666 -1669, Dallas, USA, March 2010.

[11] Y. Zigel, D. Litvak, and I. Gannot, "A method for automatic fall detection of elderly people using floor vibrations and sound - Proof of concept on human mimicking doll falls," Transactions on Biomedical Engineering, vol. 56, no. 12, pp. 2858-2867, December 2009.

[12] L. Yun, Z. Zhiling, M. Popescu, and K.C. Ho, "Acoustic fall detection using a circular microphone array," Annual International Conference of the IEEE Engineering in Medicine and Biology Society (EMBC), pp. 2242-2245, Aug. 31-Sept. 42010.

[13] M. Mercuri, D. Schreurs, and P. Leroux, "SFCW microwave radar for in-door fall detection", IEEE Topical Conference on Biomedical Wireless Technologies (BioWireless), pp. 53-56, Santa Clara, CA, USA, 15-18 Jan. 2012.

[14] M. Mercuri, P.J. Soh, L. Boccia, D. Schreurs, G.A.E. Vandenbosch, P. Leroux, and G. Amendola, "Optimized SFCW Radar Sensor Aiming at Fall Detection in a Real Room Environment", IEEE Topical Conference on Biomedical Wireless Technologies (BioWireless), pp. 4-6, Austin, TX, USA, 20-23 Jan. 2013.

[15] M. Mercuri, D. Schreurs, and P. Leroux, "Optimised waveform design for radar sensor aimed at contactless health monitoring," IET Electronic Letters, vol. 48, no. 20, pp. 1255-1257, Sep. 2012.

[16] P. Karsmakers, T. Croonenborghs, M. Mercuri, D. Schreurs, and P. Leroux, "Automatic in-door fall detection based on microwave radar measurements," European Microwave Week, Amsterdam, The Netherlands, pp. 202-205, Oct. 28-Nov. 22012. 


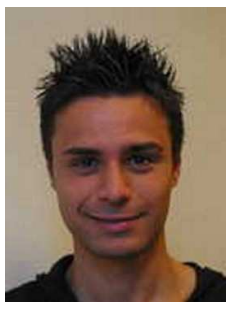

Marco Mercuri was born in Calabria, Italy in 1985 $\mathrm{He}$ received the Bachelor and Master degrees in electronic engineering from the Università della Calabria (UNICAL), Arcavacata di Rende, Italy, in 2006 and 2009 , respectively. He is currently working towards the Ph.D degree within the TELEMIC research group of the Department of Electrical Engineering (ESAT), KU Leuven, Belgium.

His research interests include biomedical applications of microwave/RF, wireless sensors, and microwave/millimeter-wave measurements.

Mr. Mercuri is a student member of the IEEE Microwave Theory and Techniques (IEEE MTT-S) and the IEEE Engineering in Medicine and Biology Society.

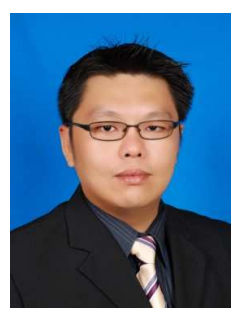

Ping Jack Soh was born in Sabah, Malaysia. He received the Bachelor and Master degrees in Electrical Engineering from Universiti Teknologi Malaysia (UTM), Johor, Malaysia, in 2002 and 2005, respectively.

From 2002 to 2004, he was a Test Engineer working on new products' test definition for manufacturing purposes. In 2005, he joined Motorola Technology Malaysia as a R\&D Engineer. There, he worked on the characterization and testing of new twoway radio antennas and RF front-ends. In 2006, he joined the School of Computer and Communication Engineering, Universiti Malaysia Perlis (UniMAP) as a Lecturer, and since 2011, a Senior Lecturer. He is currently on study leave and working towards his Ph.D in the ESAT-TELEMIC Research Division, Katholieke Universiteit Leuven, Belgium. His research interests include planar antennas, flexible/textile antennas, on-body communication, metamaterials, passive microwave components and microwave measurements

Mr. Soh was the recipient of the CST University Publication Award in 2011 and 2012, the IEEE Antennas and Propagation Society (AP-S) Doctoral Research Award in 2012, and the IEEE Microwave Theory and Techniques Society (MTT-S) Graduate Fellowship for Medical Applications in 2013.

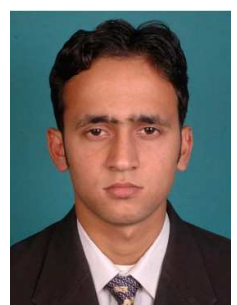

Gokarna Pandey was born in Karikot Syangja, Nepal in 1985. He received B.E. in Electronics and communication engineering from Pokhra University, Pokhara, Nepal in 2007 and M.Sc.in Electrical Engineering from KU Leuven, Heverlee, Belgium in 2012.

From 2008 to 2009, he was an assistant Lecture in Electronics and Communication department of Lumbini Engineering College, Butwal, Nepal. Recently, he completed M.Sc. degree from KU Leuven. His research interests include the antenna and microwave circuit design.

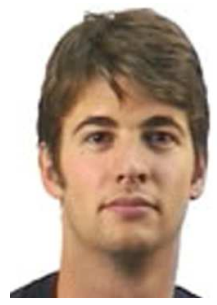

Peter Karsmakers was born on April 14, 1979. He received a M.Sc. degree in electronics-ICT engineering from the Katholieke Hogeschool Kempen (K.H.Kempen) in 2001. In 2004 he received the degree of Master in Artificial Intelligence from the Katholieke Universiteit Leuven (K.U.Leuven).

From 2004 to 2010 he was a research assistant within the SISTA research group of the Department of Electrical Engineering (ESAT), KU Leuven, Belgium. He received his Ph.D. at the K.U.Leuven in the faculty of Applied Sciences, department of Electrical Engineering in the SISTA research division in May 2010. From 2001 he combined his research activities with teaching at the Thomas More university college (formerly Katholieke Hogeschool Kempen), Geel, Belgium. He is currently a postdoctoral researcher of the MOBILAB research group of Thomas More. His main research interest are machine learning and biomedical signal processing.

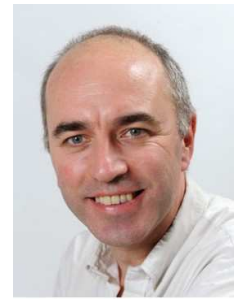

Guy A. E. Vandenbosch received the M.S. and Ph.D. degrees in Electrical Engineering from the Katholieke Universiteit Leuven, Belgium, in 1985 and 1991, respectively.

Since 1993, he has been a Lecturer, and since 2005, a Full Professor at the same university. His research interests are in the area of electromagnetic theory, computational electromagnetics, planar antennas and circuits, nano-electromagnetics, EM radiation, EMC, and bio-electromagnetics. His work has been published in ca. 150 papers in international journals and has led to ca. 250 presentations at international conferences.

Currently, he leads the Working Group on Software within the European Association on Antennas and Propagation (EuRAAP), he holds the position of chairman of the IEEE Benelux Chapter on Antennas en Propagation, and is secretary of the Belgian National Committee for Radio-electricity (URSI), where he is also in charge of commission $\mathrm{E}$

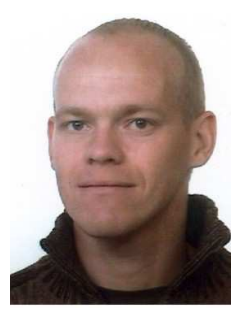

Paul Leroux (SM'10) was born in Eeklo, Belgium in 1975. He received the M.Sc. degree and Ph.D. degree in electronic engineering from the $\mathrm{KU}$ Leuven, Heverlee, Belgium, in 1999 and 2004, respectively.

From 1999 to 2004, he was a Teaching and Research Assistant within the MICAS research group of the Department of Electrical Engineering (ESAT), KU Leuven, Belgium. Since 2004 he is a professor at the Katholieke Hogeschool Kempen, Geel, Belgium, where he is head of the RELIC research group. Since 2009 he is also associated professor at the KU Leuven where he is coordinator of the Technology Cluster Electricity-Electronics-ICT of the multi-campus Faculty of Engineering Technology. His current research activities focus on radiation tolerant IC design, circuits for optical communication and instrumentation and electronics for ultra-wideband and microwave imaging systems.

Prof. Leroux has (co)authored over 80 papers in international journals or conference proceedings. In 2010, he received the SCK-CEN Prof. Roger Van Geen Award from the FWO and FNRS for his work on IC design for radiation environments.

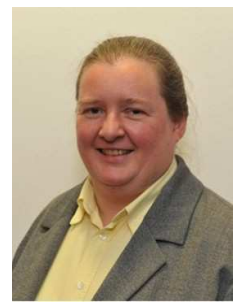

Dominique Schreurs received the M.Sc. degree and $\mathrm{Ph} . \mathrm{D}$. degree in electronic engineering from University of Leuven (KU Leuven), Belgium.

As post-doc fellow, she was visiting scientist with Agilent Technologies (USA), E.T.H. Zürich (Switzerland), and NIST (USA). She is now a Full Professor at KU Leuven. Her main research interests concern the (non)linear characterization and modelling of microwave devices and circuits, as well as (non)linear hybrid and integrated circuit design for telecommunications and biomedical applications. She is co-editor of two books, contributor to seven books, and (co-)author of about 100 journal papers and 300 contributions at international conferences.

Prof. D. Schreurs serves on the IEEE MTT-S AdCom since 2009, was vice-chair of the IEEE MTT-S Technical Coordinating Committee in 20092010, and is chair of the IEEE MTT-S Education Committee since 2012. She was the Chair of the IEEE MTT-S Technical Committee on Microwave Measurements (MTT-11) in 2005-2008. D. Schreurs is Distinguished Microwave Lecturer for the period 2012-2014. She is also Associate Editor of the IEEE Microwave and Wireless Components Letters, Associate Editor of the International Journal of Microwave and Wireless Technologies, reviewer for all IEEE MTT-S journals as well as TPRC member of IMS, RWW, and EuMW. She is also reviewer for various MTT-S (co-)sponsored conferences and regularly acts as session chair at conferences and as judge for student competitions. Beyond IEEE, Prof. D. Schreurs also serves as Technical Chair on the Executive Committee of the ARFTG organization, and initiated the NVNA Users' Forum as well as the IEEE Women in Microwaves event at the European Microwave Week. 